\title{
O Ensino de Heurísticas e Metaheurísticas na área de Pesquisa Operacional sob a ótica da Educação Dialógica Problematizadora
}

\author{
Andre Zanki Cordenonsi* \\ Felipe Martins Muller** \\ Fábio da Purificação de Bastos ${ }^{* * *}$
}

Resumo: O ensino de heurísticas e metaheurísticas na área de pesquisa operacional está centrado, basicamente, na definição de modelos matemáticos e algoritmos para o teste de teorias que são apresentadas em sala de aula. Este modelo tradicional de ensino pressupõe o professor como principal mentor do processo de transferência da teoria matemática para uma definição lógica de passos algorítmicos que os educandos devem entender e, posteriormente, implementar em alguma linguagem de programação. Neste artigo, propomos uma nova metodologia, onde seja possível consolidar uma construção participativa dos alunos através de problemas propostos em aula.

Palavras-chave: ensino de heurísticas e metaheurísticas, educação dialógica problematizadora, AMEM

\section{The Heuristics and Metaheuristics Teaching in Operational Research Considering the Problem Dialogic Education}

Abstract: The heuristics and metaheristics teaching process is centered in a set of mathematical models definitions and algorithms, which are presented in classrooms. This traditional model of education estimates the professor as a main mentor of the transference process of the mathematical theory to define algorithmical logical steps. The studens must understand the algorithms and implement it in some programming language. In this paper, we consider a new methodology to consolidate a interactive construction of the students through problems considered in the classroom.

Keywords: heuristics and metaheuristics teaching, problem dialogic education, AMEM

\section{Introdução}

A Informática na Educação tem representado uma revolução na educação tradicional, nas políticas de educação públicas e nos formatos e metodologias propostas para a interação educando - educador. No entanto, a mera adição de suportes tecnológicos à sala de aula não contribui de forma transformadora, constituindo-se, em muitos casos, de um mero acessório ou de objetos de propaganda institucionais. A grande transformação está na utilização destes recursos na criação de planos metodológicos que superem a reprodução do conhecimento e levem à sua produção. $O$ investimento tecnológico só se justifica caso o objetivo final da implementação do sistema seja plenamente satisfatório, ou seja, a efetiva aprendizagem do educando. Além disso, evidencia-se que o processo de ensino precisa mudar para um processo educativo, uma vez que, segundo Moran, "ensino e educação são conceitos diferentes. No ensino organiza-se uma série de atividades didáticas para ajudar os alunos a compreender áreas

\footnotetext{
Prof. Msc, Centro Universitário Franciscano (UNIFRA), PGIE/UFRGS, andrezc@unifra.br

"Prof. Dr. Universidade Federal de Santa Maria (UFSM), felipe@inf.ufsm.br

"* Prof. Dr. Universidade Federal de Santa Maria (UFSM), fbastos@ce.ufsm.br 
específicas do conhecimento (ciências, história, matemática). Na educação o foco, além de ensinar é ajudar a integrar ensino à vida, conhecimento e ética, reflexão e ação, a ter uma visão da totalidade". (Moran, 2000).

Neste contexto, destaca-se a prática educacional orientada pelas teorias da Investigação-Ação Educacional (IAE) e a Educação Dialógica-Problematizadora (EDP) (Carr e Kemmis, 1986). A Investigação-Ação Educacional depende de uma seqüência de julgamentos e ações que constituem as etapas do ciclo de uma espiral, com quatro fases: (a) planejamento: etapa antecessora a ação propriamente dita, onde é necessário refletir sobre a situação educativa, sua complexidade e importância, construindo uma base para as ações futuras; (b) ação: guiada pelo planejamento realizado anteriormente sem, no entanto, incorrer no erro comum de utilizar o planejamento como um guia estático e imutável. A ação deve possuir um propósito criticamente informado; (c) observação: documentação dos efeitos da ação, gerando uma base para a reflexão. A documentação contribui para a melhoria contínua da prática, através da análise da situação contextualizada, o que se pode traduzir em uma ação estratégica mais crítica; (d) reflexão: finalmente, a reflexão tenta interpretar, discursivamente, os acontecimentos oriundos das ações, propondo modificações ao planejamentos das mesmas, face às evidências observadas, reconstruindo uma nova ação informada.

Já a Educação Dialógica-Problematizadora interpreta que a ação pode ser construída metodologicamente através dos seguintes momentos (Angotti e Delizoicov, 1990): problematização inicial, organização do conhecimento e aplicação do conhecimento. A problematização inicial é apresentada como um desafio na forma de questões e/ou situações que devem ser discutidas e debatidas pelos alunos. No próximo momento, na organização do conhecimento, o educador deve orientar a sistematização do mesmo, para a completa compreensão do tema e do problema apresentado. Este conhecimento deverá ser usado pelo aluno para analisar o problema inicial e generalizálo para outras situações que são explicadas pelo mesmo conhecimento, o que caracteriza o terceiro momento, da aplicação do conhecimento. As teorias apresentadas anteriormente podem ser aplicadas na Informática na Educação através de várias metodologias, destacando-se os Ambientes Virtuais de Ensino-Aprendizagem, que podem ser utilizados como ferramentas para a eficaz implementação de métodos educacionais utilizando recursos computacionais. Este artigo apresenta uma proposta de metodologia de ensino de heurísticas e metaheurísticas, que constituem o núcleo central da área de Pesquisa Operacional, utilizando um ambiente virtual de ensinoaprendizagem cuja concepção pedagógica está focada na educação dialógicaproblematizadora. $\mathrm{O}$ estudo de caso na área de Pesquisa Operacional se justifica pela inserção desta disciplina na grande maioria dos cursos de Ciência da Computação, Sistemas de Informação e Engenharia de Computação, conforme as Diretrizes Curriculares dos Cursos da Área de Computação e Informática (MEC, 2000).

\section{Ambientes Virtuais de Ensino-Aprendizagem}

Atualmente, percebe-se um número cada vez maior de iniciativas na definição e desenvolvimento de Ambientes Virtuais de Ensino-Aprendizagem, normalmente colaborativos e baseados na Internet, onde o destaque maior está na interação entre os alunos e educadores através do ambiente. Diversas teorias pedagógicas têm sido empregadas para o norteamento destes ambientes. Para (Santos, 1999) estes ambientes são classificados como sistemas de autoria para cursos à distância (ênfase no ensino) e/ou salas de aula virtuais (ênfase no processo de ensino/aprendizagem) e, normalmente, provêem meios para a criação, participação e administração de curso usando a Internet, 
através de relações cooperativas externadas nas interações educando/educador, educando/educando e educando/material didático. Entre os diversos ambientes existentes, cita-se: (a) AulaNet (PUC-RIO): possibilita a administração, criação, manutenção e assistência de cursos à distância, com enfoque instrucionista ou construcionista(Fuks e outros, 2002); (b) Teleduc (Unicamp): possibilita a criação, participação e administração de cursos na Internet e é baseado na metodologia de formação contextualizada desenvolvida por pesquisadores do Núcleo de Informática Aplicada à Educação, da própria Unicamp (Lachi e Rocha, 2002); (c) Ensinet (UCPEL): ambiente de aprendizagem para a integração de disciplinas, agrupadas sob o mesmo tema. O Ensinet prevê a integração através de três etapas: integração através de referências mútuas, integração por entrelaçamento de atividades e integração completa de atividades (Miranda e outros, 2000); (d) Eproinfo (MEC): ambiente colaborativo de aprendizagem que permite a concepção, administração e desenvolvimento de diversos tipos de ações, como cursos a distância, complemento a cursos presenciais, projetos de pesquisa, projetos colaborativos e diversas outras formas de apoio a distância e ao processo ensino-aprendizagem. O e-ProInfo é composto por dois WebSites: o site do Participante e o site do Administrador (MEC, 2005).

\section{Projeto AMEM}

O projeto AMEM - Ambiente Multimídia para Educação Mediada por Computador na Perspectiva da Investigação-Ação Educacional, da Universidade Federal de Santa Maria, tem como objetivos o desenvolvimento de um ambiente multimídia para educação presencial, semi-presencial e à distância baseado em uma arquitetura clienteservidor e multicamadas, internet, sistemas operacionais e aplicativos freeware. A opção pela utilização de tecnologias baseadas no movimento autodenominado software livre está baseada na convicção de que a exclusão digital só será verdadeiramente combatida se todos os aspectos da mesma forem confrontados. Desta forma, a utilização de software livre multi-plataforma é crucial, pois permite que qualquer tipo de instituição, com qualquer base tecnológica instalada, possa usufruir o ambiente desenvolvido. Para tanto, foram tomadas decisões tecnológicas (banco de dados MySql, servidor Apache, linguagem de desenvolvimento PHP e JavaScript, programação sem utilização de plug-ins) que possuem versões para os principais sistemas operacionais e equipamentos existentes no mercado. As linguagens de programação PHP e JavaScript, apesar de serem mais lentas devido a interpretação, são robustas e bem estruturadas, permitindo estilos de programação eficientes e modernos.

No ambiente, os usuários podem assumir três perfis diferentes: o de educador e o de aluno, centros do processo de ensino-aprendizagem, e o de administrador, encarregado da manutenção do ambiente (Fernandèz, 2003). Existem seis módulos principais: pessoal (manutenção do cadastro dos participantes), comunicação (ferramentas para comunicação síncrona e assíncrona), disciplinas (acesso às atividades escolares), biblioteca (depósito dos materiais escolares) e ajuda (manual de uso das ferramentas do ambiente). Desta forma, o ambiente possibilita a criação, participação e administração de cursos baseados na internet de forma operacional, sem exigir do docente um amplo conhecimento das tecnologias envolvidas para disponibilizar um curso interativo através da Internet (De Bastos e Muller, 1999).

Durante a concepção do ambiente, a teoria educacional DialógicaProblematizadora foi reorganizada pelo grupo de pesquisa, e apresentada em (De Bastos e Muller, 1999), em três momentos: desafio inicial, melhor solução educacional no momento e desafio mais amplo. Inicialmente, o modelo é ativado no início da aula com um desafio inicial, que instiga os alunos a investigarem suas próprias visões de mundo V. $3 \mathrm{~N}^{\mathrm{o}}$ 1, Maio, 2005 
através de um desafio concreto e/ou um problema a ser resolvido. Através de múltiplas interações entre aluno/educador e aluno/aluno, deve-se consolidar o conhecimento científico através da codificação/decodificação da melhor solução educacional no momento, sistematizada pelo educador. Desta forma, o educador pode confrontar as visões de mundo dos alunos, tensionando as visões científicas e cotidianas, rompendo estas, e problematizando o desafio mais amplo, "que busca avaliar processualmente a universalidade, validade e limitação do conhecimento científico-tecnológico abordado na aula." (De Bastos e Muller, 1999). Deste modo, o projeto AMEM procura consolidar a problematização dos conteúdos escolares, desafiando educadores e alunos a trabalhar na escola com a perspectiva da resolução de problemas científicotecnológicos. De forma prática, o ambiente provê as seguintes facilidades (Lauermann, 2002): (a) construção da matriz dialógica-problematizadora, que possibilita a criação de uma estrutura sistemática envolvendo educador, aluno, tema de estudo e contexto, favorecendo o exame e discussão da preocupação temática; (b) implementação das atividades extra-classe, que reúne as três partes previamente definidas que compõem o planejamento: programação, atividade extra-classe e atividade de colaboração. A programação apresenta a estrutura cronológica das atividades. A atividade extra-classe é apresentada na forma de um problema a ser resolvido e a atividade de colaboração é usada pelo educador para possibilitar ao aluno participação ativa do processo de programação da próxima aula (disponibilização de bibliografias, pré-programação, recebimentos de sugestões, entre outros.); e (c) anotação de registros, onde o educador poderá registrar suas reflexões sobre cada ação.

\section{Ensino de Heurísticas e Meta-Heurísticas}

A otimização tem como objetivo a resolução da alocação de recursos, tipicamente limitados, com o intuito de alcançar determinados objetivos. Considerando que existe um conjunto discreto de soluções possíveis, a resolução de um problema de otimização combinatória inclui o processo de geração, avaliação e comparação de soluções, num determinado limite de tempo. Conforme (Corne e outros, 1999), a otimização é um tópico central nas áreas da ciência da computação, inteligência artificial e pesquisa operacional. Uma heurística pode ser definida com um algoritmo que encontra um solução factível, não necessariamente a melhor solução, para um determinado problema com uma determinada função objetivo, num tempo computacional razoável (Diaz, 1996). Estas mesmas heurísticas também podem ser facilmente adaptáveis a outros tipos de problemas envolvendo as áreas de Inteligência Artificial, Redes de Computadores e Arquitetura de Computadores. No entanto, somente a aplicação de heurísticas pode não resolver todas as classes de problemas. As meta-heurísticas, que se caracterizam por guiarem outras heurísticas, têm sido particularmente interessantes na resolução de problemas complexos. Em relação as meta-heurísticas, pode-se citar como principais métodos: simulated annealing, busca tabu, algoritmos genéticos, algoritmos meméticos e busca local genética. Apesar de extremamente relevante no que concerne a aplicabilidade, o ensino de heurísticas e meta-heurísticas é, pedagógica e tecnologicamente, normalmente apresentado de forma desinteressante e dependente da fórmula giz + quadro-negro. O ensino de heurísticas e meta-heurísticas, que usualmente apresenta um embasamento matemático forte e necessita um grande número de passos para que o processo faça sentido, torna-se desestimulante se realizado através de um processo didático tradicional. Também é importante salientar que a literatura é pobre no que concerne ao estudo didático de tais conteúdos, concentrando-se normalmente na definição e comparação de algoritmos e suas complexidades. 
Quando se fala no ensino de heurísticas e meta-heurísticas a atenção se volta, principalmente, para a modelagem, solução e análise de problemas decisórios, sendo que um estudo de caso completo corresponde à realização de experimentos numéricos com modelos lógico-matemáticos. Estes experimentos envolvem geralmente grande volume de cálculos repetitivos, fazendo-se necessário o uso intensivo do computador. Também se torna necessário o emprego de um conjunto de fórmulas e técnicas matemáticas que, se não forem ilustradas de forma aplicada, corre-se o risco de que o alcance destas não seja compreendido pelos alunos (Dávalos, 2002).

Existe um vácuo tecnológico e pedagógico que precisa ser preenchido para que o ensino das técnicas de heurísticas e meta-heurísticas se torne mais acessível para o corpo discente. Estas técnicas usualmente são discutidas dentro dos cursos de engenharia, administração e informática. No entanto, a simples exposição de cada técnica não prove ao aluno a aprendizagem significativa necessária para a real compreensão do fenômeno que está sendo investigado. Para que uma técnica seja eficientemente explorada e compreendida pelo aluno, ele precisa verificar como seu funcionamento altera as soluções encontradas no decorrer do processo, assim como os parâmetros alteram as mesmas. No entanto, as questões envolvendo a implementação das diferentes técnicas inviabilizam que todas as mesmas possam ser desenvolvidas por completo pelos alunos. Uma forma interessante proveria que o aluno testasse as técnicas e compreendesse seu funcionamento para que, posteriormente, utilizasse este aprendizado no desenvolvimento de suas próprias técnicas e funções heurísticas. Dentre as técnicas que podem ser utilizadas para o incremento da real compreensão dos alunos, a animação pode ser considerada a mais viável e eficaz. A animação por computador pode ser definida como uma sequiência temporal de mudanças visuais em uma determinada cena. Adicionando a mudança de posição dos objetos com translações ou rotações, a animação baseada em computador pode mostrar variações de tempo no tamanho do objeto, cor, transparência ou mesmo textura. A animação de sistemas dinâmicos tem sido uma técnica largamente utilizada como forma pedagógica de ensino, tais como podem ser observados nos sistemas ALMA(Varanda e Henriques, 1999) e WinHipe (Naharro-Berrocal e outros, 2001). Quando utilizada na educação, a animação pode ter duas abordagens. A primeira, denominada passiva, ocorre quando o usuário é um mero espectador da animação. Esta metodologia é comparada a experiência de assistir um filme ou uma seqüência pré-definida de eventos. Ela pode ser útil para a complementação dos aspectos vistos nas aulas presenciais, abordando somente o conhecimento pré-estabelecido pelo desenvolvedor da animação. Usualmente, sistemas que trabalham com este tipo de abordagem tem como característica principal a alta qualidade gráfica. Como exemplo, é possível citar o vídeo Warriors of the Net(Gunilla e outros, 2002), desenvolvido com o intuito de apresentar os principais conceitos das redes de computadores interligadas pela Internet. No entanto, a abordagem passiva possui uma limitação clara no que concerne a experimentação de novos conjuntos de dados, pois os parâmetros da animação são estabelecidos pelo desenvolvedor e não podem ser alterados pelos educados. Com a eliminação desta restrição e a incorporação de facilidades no manuseio das diretivas da animação, é possível para o aluno realizar suas próprias experiências, direcionando o seu conhecimento de acordo com o seu ritmo de aprendizagem. Esta abordagem é denominada ativa. Sistemas que trabalham com esta metodologia geram animações com uma qualidade gráfica mais baixa, com o intuito de não sobrecarregar a máquina. Como exemplos, é possível citar os trabalhos de (Martins e outros, 2003) e (Zachary, 2004). Do mesmo modo que a experimentação durante a operação das animações enriquece o aprendizado mais do que a mera observação passiva delas, é de se esperar que, com um sistema onde a própria 
implementação das animações gráficas é facilitada a ponto de poder ser realizada pelo estudante, a absorção do funcionamento das heurísticas seja ainda mais intensa.

\section{Ambientes de Desenvolvimento de Otimização Combinatória}

O desenvolvimento de técnicas de otimização combinatória pode ser beneficiada pelo incremento da internet por causa de dois fatores: (a) não existe uma única técnica para resolver problemas de otimização. Para um mesmo problema, métodos e tecnologias competem em termos de velocidade, custo e conveniência; (b) novas aplicações tipicamente envolvem a construção de novos modelos, que devem ser especializados para os problemas específicos.

Além disso, as novas aplicações emergentes podem exigir a confluência de diversas técnicas que, em conjunto, atingem boas soluções. Para tanto, meta-heurísticas podem ser utilizadas como guias que gerenciam a criação e o melhoramento de soluções incumbentes. O desenvolvimento de recursos via internet para a resolução de problemas de otimização é algo recentemente novo. Nas últimas décadas, houve um avanço considerável nos esforços em construir ferramentas de resolução que utilizem a internet como ponto de apoio. A maioria destes servidores tem como características comuns a resolução de problemas específicos, que precisam ser modelados em alguma linguagem e/ou instância específica para serem resolvidos. Não há como combinar métodos ou formas de resolução.

O CORE (Combinatorial Optimization Distributed Resource Management Environment), ou Ambiente de Gerenciamento de Recursos de Otimização Combinatória Distribuídos, se caracteriza por ser um ambiente baseado na estrutura da Internet que suporta a execução remota distribuída de diferentes métodos de resolução para problemas de otimização combinatória. O sistema CORE fornece um repositório de instâncias que armazena dados de teste com valores das melhores soluções encontradas para esses problemas. Com a ajuda de assistentes gráficos, os usuários podem criar planos de otimização, navegar pelo repositório de métodos e instâncias, enviar planos de otimização para a resolução em servidores remotos e receber resultados e estatísticas sobre a qualidade dos métodos utilizados(Santos, 2002). A arquitetura do sistema CORE utiliza o modelo cliente/servidor. No servidor encontra-se o repositório de problemas, métodos, instâncias e usuários, além do servidor de otimização, que disponibiliza um determinado nodo de processamento para o ambiente. No cliente estão as ferramentas administrativas, que gerenciam os recursos do repositório, e o editor de planos, que fornece assistentes gráficos para a criação de planos de otimização utilizando os algoritmos existentes no servidor. Toda a estrutura do sistema CORE foi concebida para fornecer ao usuário um modelo padrão para heurísticas e metaheurísticas, baseado nos conceitos de design patterns e programação genérica, utilizando a linguagem Java. Desta forma, foi desenvolvido um framework genérico para construção de métodos heurísticos que apresenta implementações de funções comuns a todos os métodos: leitura dos dados iniciais, apresentação dos resultados, implementação de algoritmos exatos, entre outros.

O CORE poderá ser utilizado como um repositório das soluções encontradas pelos alunos, fornecendo aos mesmos uma série de facilidades no desenvolvimento rápido de seus próprios algoritmos, condição imprescindível para a compreensão da área de heurísticas e meta-heurísticas. Além disso, como parte deste trabalho, pretende-se ampliar o sistema de forma a conter representações mais didáticas para a visualização dos dados científicos, através de gráficos e execuções controladas pelo usuário. As principais vantagens do CORE podem ser definidas como: (a) geração de planos de 
otimização, que podem combinar diferentes técnicas na mesma resolução; (b) definição de um framework de implementação de novos métodos. $\mathrm{O}$ ambiente CORE define um meta-modelo para que os novos métodos, exatos, heurísticos ou meta-heurísticos, possam ser implementados com um menor esforço de programação e, principalmente, inseridos no sistema sem a necessidade de adaptações ou pré-processamentos que, invariavelmente, influenciam no desempenho geral do sistema.

\section{Metodologia Proposta}

A metodologia proposta para o ensino de heurísticas e metaheurísticas baseia-se nos três momentos dialógico problematizadores. Inicialmente, era realizado um pacto entre educando e educador, onde se apresentava ao aluno a metodologia proposta e a realização das avaliações através de projetos, sem a utilização de provas formais. Os alunos deveriam se comprometer em conduzir os desafios propostos com responsabilidade e utilizar os seminários de acompanhamento como forma de verificação dos trabalhos. Ao final, um artigo científico deveria ser apresentado como relatório final.

Uma característica importante da metodologia proposta é a condução da disciplina através de um grupo de educadores, denominados P1, P2, ... Pn. Este grupo decide sobre as estratégias a serem abordadas em cada aula, assim como os facilitadores pedagógicos (tecnológicos ou não) que poderiam ser utilizados em cada encontro. Normalmente, um grupo de educadores é formado pelo professor responsável pela disciplina e seus monitores provenientes da graduação ou alunos da pós-graduação em docência orientada. A primeira tarefa a ser construída pelo grupo de educadores é definição de um projeto final, ou tema, onde o desenvolvimento da disciplina será baseado. No caso do ensino de heurísticas e metaheurísticas, é proposto que o projeto seja baseado em um problema clássico da literatura, onde diversas metodologias podem ser apresentadas e testadas pelos alunos.

Desta forma, as aulas seguem um esquema básico, que pode ser alterado pelos educadores de acordo com o andamento da disciplina:

- através do ambiente AMEM, o material relacionado à aula (algoritmo e/ou método matemático a ser discutido) é disponibilizado com, pelo menos, uma semana de antecedência, através da ferramenta programação, onde o grupo de educadores define o dia e a hora do encontro presencial, assim como as atividades que serão desenvolvidas dentro desta. O material, seja ele digital ou apenas um apontamento para uma bibliografia, pode ser consultado pelos alunos e, eventualmente, o educando também pode enviar comentários para os professores acerca do assunto a ser debatido na aula subseqüente. No ambiente AMEM, esta atividade é denominada atividade de colaboração e é sempre realizada antes da aula presencial;

- no início da aula presencial, o grupo de educadores apresenta um problema para os educandos. Este é o primeiro momento, denominado desafio inicial. No caso específico, é aconselhável que se apresente uma instância de um problema real de otimização, cuja solução seja conhecida e que o tempo gasto pelos grupos de educandos para resolver o problema seja viável com a disponibilidade de tempo da aula presencial. Além disso, é interessante que a escolha do problema seja feita com cuidado, para que o educando possa testar os algoritmos clássicos e suas próprias idéias de forma satisfatória. Por exemplo, numa aula inaugural do curso, é possível apresentar um problema de caixeiro viajante onde o algoritmo clássico de buscar sempre a cidade mais próxima apresente resultados bastante 
insatisfatórios. A realização dos cálculos numa base real para idéias básicas de otimização tendem a expandir os conceitos dos educandos, que percebem a necessidade de buscar novos métodos, frente a pouca robustez dos algoritmos mais simples;

- após este trabalho, realizado normalmente em grupos, os educadores recolhem os resultados dos alunos, materializados em algoritmos/métodos de resolução e resultados possíveis, formando o segundo momento, denominado melhor solução educacional no momento. As soluções/algoritmos/métodos são comparadas e comentários são realizados sobre o pensamento que norteou estas determinadas soluções. Após esta discussão, os algoritmos clássicos, previamente definidos pelo grupo de educadores, são apresentados e discutidos com os alunos. Neste estágio, é importante salientar os aspectos similares ou contraditórios dos algoritmos clássicos com os algoritmos desenvolvidos pelos alunos, como forma de conduzir o trabalho de forma contínua, além de valorizar as idéias e estimular o pensamento criativo dos educandos;

- finalmente, o grupo de educadores pode construir o terceiro momento, denominado desafio mais amplo. No caso do ensino de heurísticas e metaheurísticas, este momento pode ser utilizado de duas maneiras: a) como forma de consolidar o conteúdo desenvolvido, através da apresentação de um problema mais complexo ou diferente, mas que possa ser resolvido através das idéias iniciais apresentadas na aula presencial; b) como forma de realizar uma ligação com o próximo conteúdo, onde um problema proposto só pode ser resolvido através da leitura e compreensão do material disponibilizado para a próxima aula. Independente da forma utilizada, o ambiente AMEM fornece suporte a este momento através da disponibilização de uma ferramenta denominada atividade extra-classe, onde o grupo de educadores pode inserir as questões relativas a esta atividade.

Algumas questões importantes são levantadas ao se utilizar esta metodologia:

- o tempo gasto na preparação das aulas é substancialmente maior, principalmente na definição dos problemas, que precisam ser escolhidos com cuidado e cujos resultados devem ser bem conhecidos;

- o grau de conhecimento do grupo de educadores também deve ser mais consolidado, pois os alunos estarão interagindo mais fortemente com os mesmos, propondo novas soluções e testando continuamente a forma como os métodos se comportam. Além disso, a fase da melhor solução educacional no momento deve ser enriquecida com os comentários acerca dos métodos desenvolvidos pelos próprios alunos, o que é realizado em um tempo bastante exíguo, exigindo uma preparação bastante acentuada de todos os membros do grupo de educadores;

- a utilização do ambiente AMEM como ferramenta de trabalho é extremamente importante, principalmente pela direção que é dada pelo sistema, que apresenta uma aula já previamente focalizada nos três momentos dialógicoproblematizadores. No entanto, deve-se tomar cuidado em não utilizá-lo como simples aparato tecnológico ou página para descarga de material didático. 


\section{Conclusões}

A metodologia proposta, baseada na Educação Dialógico-Problematizadora, se mostrou bastante adequada ao ensino de heurísticas e metaheurísticas, pois a área de pesquisa operacional é, essencialmente, problematizadora, sendo que a metodologia têm sido aplicada nas disciplinas da Universidade Federal de Santa Maria, com bastante sucesso. Nota-se um crescente interesse dos educandos pela disciplina, além de um bom grau de amadurecimento no conteúdo desenvolvido. Pelas experiências realizadas, é consenso que este amadurecimento foi atingido principalmente por causa das discussões realizadas depois da apresentação do desafio inicial, durante a fase da consolidação do conhecimento pelo grupo de educadores. Os educandos apresentaram questionamentos em maior número e de qualidade superior, evidenciando um maior conhecimento sobre os assuntos tratados.

Um aspecto importante, tanto do ambiente quanto da metodologia, é que o educador, ou grupo de educadores, continua sendo essencial para o desenvolvimento das aulas. Apesar de não ser o centralizador do conhecimento, pois ele já o partilha desde o início, deixando que os próprios alunos possam construí-los e desenvolvê-los a partir de suas idéias individuais e coletivas, o professor deve dirigir as aulas, conduzindo os alunos ao conteúdo que está sendo desenvolvido. Desta forma, há espaço para discussão e debates de idéias novas e, potencialmente, profícuas, mas também há a necessidade de se consolidar um determinado conteúdo que faz parte de uma disciplina de graduação. Através desta metodologia, é possível melhorar o entendimento dos métodos matemáticos envolvidos na área de pesquisa operacional sem perder a profundidade necessária.

\section{Referências}

Angotti, J. A. P. e Delizoicov, D. N. Metodologia do Ensino de Ciências. São Paulo: Cortez, 1990.

Carr, W., Kemmis, S. Becoming Critical: Education, Knowledge and Action Research. Brighton, Uk: Falmer Press, 1986.

Corne, D., Dorigo M., Glover, F. New Ideas in Optimization. Mcgraw Hill, Berkshire, 1999.

Dávalos, R.V. Uma Abordagem do Ensino de Pesquisa Operacional Baseada no Uso de Recursos Computacionais. In: International Conference On Engineering And Technology Education. 6. Proceedings... Santos. Brasil, 1999.

De Bastos, C.; Muller, F.M. Criando Desafios em Informática. In: Atas Da Iv Escola De Verão Sobre Investigação-Ação Educacional. UFSM, Santa Maria, RS, 1999.

Diaz, A. Optimización Heurística Y Redes Neuronables. Editorial Paraninfo, Madrid, 1996.

Fernandèz, E.G. Ambiente Multimídia Para Educação Mediada Por Computador: Modelagem e Implementação. Dissertação de Mestrado. Ppgep, UFSM, 2003.

Fuks, H., Raposo, A.B., Gerosa, M.A. Engenharia De Groupware: Desenvolvimento De Aplicações Colaborativas. Anais Do Xxii Congresso Da Sociedade Brasileira de Computação. V.2, 2002.

Gunilla, E., Stephanson, T., Hanberger, N. "Warriors Of The Net". Vídeo. Disponível por www em http://www.warriorsofthe.net. Acesso em abril de 2004, 2002. 
Lachi, R.L., Rocha, H.V. Uso De Agentes De Interface Para Auxiliar A Avaliação Formativa No Ambiente Teleduc. In: Xiii Simpósio Brasileiro De Informática Na Educação. Anais, São Leopoldo, 2002.

Martins, A.J. Fiolhais,C E Paiva, J. Simulações On-Line no Ensino de Física e Química. Revista Brasileira De Informática Na Educação. Numero 7. Sbc. P.111117. V. 11 - N. 2. Julho/Dezembro, 2003.

MEC, Diretrizes Curriculares dos Cursos da Área de Computação e Informática. Ministério da Educação e Cultura. Disponível por www em: http://www.mec.gov.br/sesu/ftp/curdiretriz/computacao/co_diretriz.rtf, 2000.

MEC. Conheça o e-proinfo. Ministério da Educação e Cultura. Disponível por www em: http://www.eproinfo.mec.gov.br/fra_eProinfo.php?opcao=1. 2005.

Miranda, R.M., Dimuro, G.P., Costa, A.C.R. Ensinet: Um Ambiente De Suporte Ao Ensino Integrado Dos Fundamentos Matemáticos da Computação. In: Anais Do Xxiv Congresso Nacional De Matemática Aplicada E Computacional, 2000.

Moran, J.M. Ensino e Aprendizagem Inovadores com Tecnologias Audiovisiuais e Telemáticas. In: Novas Tecnologias Pedagógicas. Moran, Masetto, Behrens (Eds). Campinas, São Paulo, 2000.

Naharro-Berrocal, F., Pareja-Flores,C.J. Velázquez-Iturbide, A. E Martínez-Santamarta, M. Automatic Web Publishing Of Algorithm Animations. Informatique Visualization Of Software. N.2 - April, 2001

Santos, H.G. Ambiente De Utilização e Gerenciamento De Recursos De Otimização Combinatória Distribuídos, Dissertação De Mestrado. PPGEP, UFSM, 2002.

Santos, N. Estado Da Arte Em Espaços Virtuais de Ensino e Aprendizagem, Revista Brasileira De Informática Na Educação. N.4. Abril Disponível por www em http://gmc.ucpel.tche.br/rbie-artigos/nr4-1999/070tu-santos.htm, 1999.

Varanda, M.J. E Henriques, P.R. Animação De Algoritmos Tornada Sistemática. Actas Do $1^{\circ}$ Workshop Computação Gráfica Multimédia E Ensino, 1999.

Zachary, J.L. Introduction To Scientific Programming Computational Problem Solving Using: Maple And C". University Of Utah. Animations Available from http://www.cs.utah.edu/ zachary/isp/applets/cannon/ cannon.html e http://www.cs.utah.edu/ zachary/isp/applets/slidingblock/ slidingblock.html. 1999. 